\title{
EL TRABAJADOR SOCIAL EN EL TRABAJO DE GRUPO
}

ESTHER VILLEGAS CASTRILLO

Profesora de Trabajo Social

EUTSA

\section{DELIMITACION DEL TEMA Y CRITERIOS ORIENTADORES}

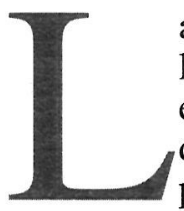

a finalidad de este estudio consiste en exponer cuáles son las funciones del Trabajador Social en el trabajo de grupo, entendido éste como forma y método de Trabajo Social que ayuda, de diversos modos, a la solución de problemas personales, o de grupo, o de organización y de promoción del bienestar social.

En primer lugar haremos referencia a algunos presupuestos que están a la base del tema específico elegido y que condicionan el modo de abordarlo y desarrollarlo.

Se trata, en particular, de los diversos modelos de práctica de trabajo de grupo, de la estructura, funcionamiento y desarrollo del pequeño grupo, y del contexto social en que desarrolla su labor el Trabajador Social en el trabajo de grupo.

El cuerpo central del estudio está constituido por el análisis de las tareas que competen al Trabajador Social en cuanto miembro y líder de grupo. Después de exponer brevemente cuál es el sentido y alcance que tiene la presencia del Trabajador Social en las diversas fases del proceso grupal, dedicaremos un desarrollo más amplio al estudio de algunas claves del rol de líder que desempeña en él, prestando especial atención a las situaciones de conflicto que pueden plantearse.

Suponemos conocidos los diversos modelos de práctica de trabajo de grupo, de acuerdo con los objetivos que motivan su formación y los métodos de que sirven para hacerlos efectivos.

En este sentido, considero orientadora, por ejemplo, la clasificación que propone A. Brown. Yendo más allá de la distinción que hacen Pappel y Rothman ${ }^{1}$ entre los modelos que denominan terapéutico, recíprocos y de objetivos sociales, esto es, centrados respectivamente en

1 Pappel, C. y Rothman, B., Relating the Mainstream Model of Social Work with Groups to Group Psychotherapy and the Structured Group Approach, SWG, 3 (2), 1980. 
la adaptación social del individuo, la mediación entre los individuos y la sociedad y la promoción efectiva de la justicia social, distingue una serie de categorías que, en síntesis, son éstas:

- Grupos que se forman para valorar necesidades, habilidades o comportamientos individuales que pueden constituir un apoyo importante en intervenciones posteriores.

- Grupos que ofrecen apoyo a personas que han de afrontar situaciones y circunstancias personales o sociales difíciles, como es el caso de disminuidos físicos o discapacitados.

- Grupos que se orientan a favorecer el cambio individual de diferentes modos y que abarcan una amplia gama de actividades relacionadas con el control social, el comportamiento interpersonal, las circunstancias materiales de dificultad o los problemas del crecimiento y desarrollo de las personas.

- Grupos centrados en tareas de educación y formación en diversos campos de asistencia y servicio a la comunidad, o que realizan una labor de mediación entre los individuos y los sistemas sociales, o que colaboran con grupos naturales ya existentes.

- Finalmente, grupos que tienen como objetivo promover cambios sociales, o aproximar la Administración a las gentes, o lograr una distribución más coherente y operativa de los sistemas institucionales.

Aunque el término trabajo de grupo incluye de una forma poco precisa una gran variedad de enfoques, y los modelos y métodos pueden agruparse de formas diferentes, hay procesos básicos y técnicas específicas que son aplicables a la mayoría de los grupos y que pueden ser adoptadas por los profesionales a los diversos contextos en que actúan, a los distintos estilos de trabajo y de intervención para solucionar problemas concretos ${ }^{2}$.

En segundo lugar, es útil tener presente la concepción del pequeño grupo como sistema social, tal como ha expuesto, por ejemplo, Louise C. Johnson en su libro Social Work Practice ${ }^{3}$.

En efecto, como todos los sistemas sociales, los grupos tienen una estructura, un modo de funcionamiento en orden a realizar tareas específicas y un desarrollo concreto a través de una serie de etapas que van sucediéndose en el tiempo.

Aunque a lo largo de nuestra exposición haremos referencia explícita a algunas de las ideas de esta autora, pienso que puede ser

2 Brown, A., Treball de Grup, Ed. Portic, S.A., Barcelona, 1988, págs. 25 y ss.

${ }^{3}$ Johnson, L. C., Social Work Practice. A Generalist Approach, Library of Congress Catalogin in Publication Data, Londres, 1986, págs. 197-228. 
orientador el esquema en que sintetiza su posición y que, reducido a sus formulaciones esenciales, es el siguiente:

\section{EL PEQUEÑO GRUPO COMO SISTEMA SOCIAL}

\section{Estructura:}

II. Funcionamiento:
A) Límites
B) Marco de relaciones
C) Vínculos
A) Equilibrio/Estabilidad
B) Toma de decisiones
C) Comunicación
D) Ejecución de tareas

III. Desarrollo:
A) Identificación
B) Factores

\section{Resistencias y límites}

Finalmente el estudio ha sido pensado y estructurado teniendo presente el contexto histórico-social y cultural en que el Trabajador Social realiza actualmente su labor específica.

Porque antes del Trabajo Social como profesión, está el Trabajo Social entendido en sentido amplio, esto es, como intento de organización racional de la sociedad, tal como ha venido realizándose a través de ese proceso complejo y ambivalente que llamamos modernidad ${ }^{4}$.

Entre sus aspectos más negativos hay que señalar el sometimiento de los individuos al trabajo del experto, la homogeneización de las conductas y el papel creciente que fue adquiriendo el Estado a través de su labor tutelar omnipresente.

Los años setenta han sido la ocasión de una rica floración sociológica que ha ilustrado de forma convincente el control social ejercido por todas las profesiones que, en nombre del bienestar de los usuarios, no han cesado de invadir el terreno de la vida cotidiana. En el corazón de este dispositivo, una ideología confusamente formulada y que se pretende liberadora ha servido para justificar esta labor de domesticación que busca conseguir la participación de los individuos en las instancias institucionales de planificación del bienestar.

4 Un desarrollo razonado de estas ideas puede verse en Renaud, G., «Travail Social, Crise de la Modernité et Post-Modernité», Canadian Social Work Review, vol. 7, núm. 1, 1990, págs. 27-48; refiriéndose a las ideas expuestas por Melucci a propósito de los Movimientos Sociales Contemporáneos, el autor resume su planteamiento con estas palabras «les mouvements sociaux (lorsqu' on les définit comme expression d'un malaise et d'une demande sociales) expérimentent le désenchantement face à la politisation et à l'institutionnalisation de ces demandes par ceux-là mèmes parfois qui s'en réclament». 
Partiendo de esta constatación, es lógico el esfuerzo de quienes optan por el Trabajo Social, entendido como profesión y modo de vida, para superar las posiciones estrictamente técnicas e infundir nuevos valores y criterios en las relaciones humanas. En particular, sus intervenciones se inspiran más en la diferencia y en la personalización que en el culto inconsciente de la identidad uniforme y homogénea, superando la pretensión de reducir su trabajo profesional a lo estrictamente técnico.

En este sentido, se tienen presentes aquellas orientaciones de las corrientes sociológicas que rechazan el punto de vista, mantenido en otro tiempo, de que la realidad colectiva determina unilateralmente el comportamiento humano. Todas ellas piensan que son los individuos lo que construyen la realidad social, atribuyendo a la interacción una serie de cualidades positivas.

\section{EL TRABAJO SOCIAL COMO MIEMBRO Y LIDER DE GRUPO}

\subsection{Observaciones preliminares: El Trabajador Social en las di- versas fases del proceso grupal}

Al abordar la exposición de las funciones del Trabajador Social en el grupo y los roles que desempeña en él en orden a su desarrollo interno y a la efectividad de sus tareas específicas, tenemos presente lógicamente aquellas técnicas o actuaciones que se consideran válidas para cualquier tipo de grupo. Porque es evidente de que cada uno de ellos en particular, con unos miembros y unos objetivos concretos exige también unas técnicas y unas actividades específicas.

Por otra parte, es evidente que, en cuanto miembro y líder de grupo, las funciones del Trabajador Social no pueden entenderse sino en relación con las diversas fases y procesos que se desarrollan en él y que condicionan tanto las relaciones internas como su modo de afrontar sus tareas y responsabilidades.

Por ello, antes de referirnos a las funciones más directamente relacionadas con el rol de líder del Trabajador Social en el trabajo de grupo, haremos una referencia más genérica al sentido que tiene su presencia en las diversas fases del proceso grupal, favoreciendo la participación, la intercomunicación o la integración, y siendo, en suma, el animador y dinamizador de la vida interna y de la actividad específica del grupo.

Y hacemos esto teniendo presente que ese proceso, en sus líneas generales, se da en cualquier grupo, aunque cada uno de ellos tiene lógicamente su dinámica propia.

En esta tarea son fundamentales las aportaciones realizadas por 
diversos autores, entre los que destaca, por ejemplo, la labor pionera de Tuckman o la de Garland y sus colaboradores ${ }^{5}$, según los cuales pueden distinguirse las fases siguientes:

1. ${ }^{\text {a }}$ Fase de formación, que fenomenológicamente está ligada a las expectativas y a la situación de punto de partida de cada miembro, suscitando lógicamente temores y recelo ante lo nuevo, ya sea la formación misma del grupo, o las tareas a realizar, o la discusión de los programas y métodos, a la vez que se esperan efectos positivos de la organización del grupo y de su funcionamiento.

Los psicólogos han subrayado que las primeras relaciones, tanto entre los miembros del grupo como con sus animadores, son una fuente constante de sorpresa, actualizándose en esos momentos todos los modelos de relación aprendidos y practicados por cada uno en otros grupos primarios anteriores, como la familia o el ambiente social ${ }^{6}$.

La etapa de formación del grupo estará también influida por el hecho de que alguno o todos los miembros se conozcan y hayan tenido contactos previos de diversa naturaleza. En cualquier caso las intervenciones del animador en esta fase inicial deberán orientarse a facilitar un clima en que la expresión de cada uno pueda darse, aportando información clarificadora sobre la tarea que se pretende e introduciendo una dinámica motivadora y de trabajo conjunto. En este sentido, los miembros del grupo deben ir adquiriendo la certeza de que su progreso depende de la aportación de todos y de cada uno, de sus participación activa en la toma de decisiones y en la ejecución de las diferentes tareas. Lógicamente, en esta etapa se hacen más patentes las diferencias individuales entre los miembros, por lo que debe ofrecerse un apoyo más directo a los que parezcan más débiles o descompensados, partiendo de la convicción de que la clave está en introducir ya desde el principio, una dinámica que fomente la cohesión y el trabajo conjunto.

Normalmente, esta fase concluye con ciertos reajustes necesarios para una mayor eficacia en las tareas. Esos reajustes deben ser vividos por el grupo como indicadores de que el proceso participativo es una realidad y que se refieren a normas concretas de funcionamiento, a aspectos organizativos y a tomas de decisión que interesan a todos.

5 Tuckman, B. W., Development Sequence in Small Groups, Psychological Bulletin, 63 (6); Garland, J. A.; Jones, H. E. y Kolodny, R. L., A. Model for Stages of Development in Social Work Groups; a S. Berstein (ed.), Explorations in Group Works, Boston University School of Social Work, 1965.

6 Una breve exposición de la situación grupal y sus consecuencias en esta fase inicial, puede verse en el trabajo de Arrieta Olmedo, L., «Los procesos de reinserción de los grupos marginados», Documentación social. Revista de estudios sociales y de sociología aplicada. Métodos de intervención social, núm. 81,1990 , págs. 119 y ss. 
Allan Brown subraya el hecho de que en la primera reunión de un grupo la tarea del Trabajador Social no tiene componentes básicos precisos, variando la importancia de éstos y el orden cronológico según los diversos tipos de grupo ${ }^{\text {? }}$.

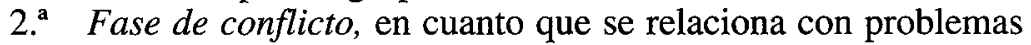
de poder y control y que se considera una fase crítica en el desarrollo del grupo, esto es, puede significar su éxito o fracaso. Los miembros del grupo comienzan a buscar su rol y su espacio propios pudiendo producirse tensiones o reacciones que propicien el enfrentamiento. A los temores iniciales de no sentirse integrado, puede suceder el temor contrario de absorción y de pérdida de su significado individual.

En cuanto a la tarea a realizar, puede surgir un sentimiento de angustia o incertidumbre, a causa de la perplejidad en cuanto al modo de proceder y de participar en las actividades programadas por el grupo. En situaciones de esta naturaleza, es evidente la necesidad de que el animador manifieste al mismo tiempo seguridad y flexibilidad, con el fin de evitar situaciones que pudieran bloquear el proceso de formación en marcha.

3. ${ }^{\mathrm{a}} \quad$ Fase posterior de búsqueda de cohesión y de profundización de las relaciones entre los miembros del grupo, llamada también fase normativa, en cuanto que se establecen normas o criterios de hacer las cosas que hacen posible llegar a un acuerdo sobre lo que está autorizado, y sobre los límites qe no deberán sobrepasarse.

En el plano emocional se constata una mayor aproximación entre los miembros del grupo, haciéndose más fácil la solución de los posibles conflictos que vayan surgiendo. Todo ello favorece la labor propia del coordinador y dinamizador de fomentar la participación voluntaria de todos en la realización de las tareas acordadas. En expresión de A. Brown, esta etapa marca con frecuencia una línea divisoria en la vida del grupo ${ }^{8}$.

Refiriéndose a estos momentos de autorregulación del funcionamiento del grupo, los teóricos de la dinámica de grupos ${ }^{9}$ han descrito con precisión las características que diferencian entre sí a los grupos maduros e inmaduros. Mientras que en estos últimos es evidente la pobreza de comunicación y de organización, así como la poca claridad de los roles y metas, todo lo cual origina una insuficiente cohesión y falta de participación, en los grupos maduros la situación es diferente: existe un clima favorable a la expresión de sentimientos y opiniones; los propósitos y objetivos se hacen más explícitos; se faci-

7 Brown, A., op. cit., pág. 91

8 Ib., págs. 94-95.

9 Cf., por ejemplo, Maisoneuve, J., La dinámica de grupos, Nueva Visión, Buenos Aires, 1980, págs. 75 y ss.; Tschorne, P., La Dinámica de grupo aplicada al Trabajo Social, Ed. Obelisco, Barcelona, 1990, pág. 20. 
lita la integración de los valores y metas individuales con las que son específicas del grupo, lo cual propicia una participación activa en las responsabilidades comunes.

4. ${ }^{\mathrm{a}} \quad$ Fase de trabajo, esto es, aquélla en la que el grupo utiliza sus capacidades y el potencial de sus miembros para conseguir sus objetivos y buscar una solución efectiva a los problemas planteados. En algunos grupos, es el momento en que se alcanza la verdadera autonomía y puede hablarse realmente de su propia identidad.

5. Por último, hay una etapa final, la de la disolución del grupo, y que puede deberse a diversas razones: por ejemplo al cumplimiento de las metas que se había fijado, al cambio de los miembros que lo componen, a la falta de apoyo institucional o económico, a la ruptura de los vínculos, o al abandono de los valores comunes y normas básicas de actuación.

Es evidente que la labor del dinamizador o líder en relación con los miembros del grupo y sus sentimientos varía según las causas de la disolucion, esto es, según pueda hablarse de fracaso o de cumplimiento parcial o total del programa previsto. En cualquier caso son situaciones que deberán estar previstas desde el comienzo de la formación del grupo, así como las soluciones que deberán darse a ellas.

\subsection{El Trabajador Social como líder de grupo}

Tras estas observaciones sobre las diversas fases del proceso grupal, es ya posible hacer referencia explícita a la labor del Trabajador Social como líder del grupo.

El liderazgo, como expone convincentemente M. Shaw, constituye uno de los roles más importantes asociados a la posición del miembro dentro de la estructura grupal y ha sido estudiado más ampliamente que cualquier otro rol mediante múltiples técnicas de investigación ${ }^{10}$.

En su definición han influido distintos puntos de vista. En unos casos se insiste en el hecho de que el líder constituye el punto focal de la conducta del grupo. En otros se define el liderazgo en relación con los objetivos grupales, viendo en el líder la persona capaz de conducir al grupo hacia sus objetivos. Algunos autores prefieren ver en el líder aquella persona que nombran como tal los miembros del grupo. Otro enfoque es el de quienes prefieren definirlo como la persona que tiene una influencia demostrable sobre la llamada sintalidad del grupo, es decir sobre el rendimiento mensurable del mismo. Más

10 Shaw, M. E., Dinámica de Grupo. Psicología de la conducta de los pequeños grupos, Biblioteca de Psicosociología núm. 7, Herder, Barcelona, 1986, pág. 310 . 
arraigo ha tenido otra definición inspirada en un criterio operativo y pragmático según la cual el líder es la persona que desarrolla conductas de liderazgo.

Al estudiar las funciones del Trabajador Social en el grupo, entiendo que es preferible atenerse a la sugerencia que hace A. Brown de utilizar el término líder en un sentido más concreto para designar aquella persona o personas a quien ha sido asignado ese rol. De hecho, efectivamente, en la mayoría de los grupos formados al Trabajador Social se le asigna ese rol, esto es, se le considera la persona central del grupo ${ }^{11}$.

\subsubsection{Algunas claves del rol de líder en el trabajo de grupo}

Más en particular, y a fin de entender las funciones que competen al Trabajador Social como líder del grupo pienso que es conveniente tener presente la distinción que se viene haciendo entre liderazgo de tarea y liderazgo de mantenimiento de grupo. Las funciones del primero se relacionan con los objetivos del grupo y sus actividades, lo cual supone la búsqueda de información, la capacidad de orientación, de organización, de diagnóstico, análisis y coordinación. En cuanto al liderazgo de mantenimiento, sus funciones se dirigen básicamente a propiciar relaciones satisfactorias en el seno del grupo, favoreciendo así la participación, el alivio de las tensiones, la existencia de un clima emocional positivo y de una comunicación que fomenta la confianza, vigoriza al grupo y ayuda a resolver las tensiones interpersonales.

Cuando se habla de técnicas de realización de la tarea se está haciendo referencia a aquellas que se relacionan con las funciones de liderazgo, muchas de las cuales tienen que ver con el desarrollo de los programas que el grupo elabora para realizar determinados objetivos y que incluyen, por tanto, decisiones sobre la estructura, la planificación y las funciones concretas a realizar. Otro conjunto de técnicas de tarea se relacionan con la valoración y análisis del grupo, la evaluación, el seguimiento y la elaboración de informes. Tareas complementarias serían también las que se relacionan con el establecimiento de contactos, personas y organizaciones, para obtener recursos o garantizar unas condiciones externas favorables a la labor del grupo.

En esta labor, es evidente que el Trabajador Social que es miembro efectivo de un grupo está especialmente cualificado para desempeñar un rol muy positivo en orden a su funcionamiento. Louise Johnson resume su aportación en las tareas siguientes:

11 Brown, A., op. cit., pág. 65. 
Ante todo, la labor de consulta, que el Trabajador Social realiza ofreciendo información y consejos, teniendo siempre presente la situación que vive el grupo, el tipo de clientes a que presta sus servicios y las alternativas existentes para el desarrollo de planes eficaces de acción.

Es también importante la tarea de facilitación de las actividades del grupo y de los procesos que se viven en él. En esta labor puede ser eficaz la descripción del funcionamiento de otros grupos, la capacidad para centrar los problemas o proceder a su discusión y el recurso a medios apropiados para aliviar las tensiones.

Finalmente, esa colaboración activa se concreta en la atención permanente a la coordinación de las actividades que realizan los miembros del grupo, a fin de que se cumpla el plan previsto sin alteraciones o rupturas de ritmo provocadas por la ineficacia de los métodos o el recurso o la improvisación ${ }^{12}$.

Para entender los roles del dinamizador del grupo en relación con los objetivos que se propone y las tareas que realiza, entiendo que es útil el siguiente cuadro que inspirándose en William Underwood ha elaborado recientemente $\mathrm{P}$. Tschorne ${ }^{13}$ sobre los roles orientados hacia el trabajo de dinamizador; tales roles son:

\begin{tabular}{cc} 
Inhibidores por & Inhibidores por \\
escasez de & Facilitadores \\
comportamiento & comportamiento \\
\hline
\end{tabular}

No iniciar ideas cuando se necesitan

Permitir que el problema se estanque cuando se necesita nueva información

No pedir opiniones a los demás cuando podrían ser útiles

Retener información cuando se necesita

Retener elaboración
Iniciar nuevas ideas

Sugerir o proponer nuevas actividades

Buscar información

Pedir clarificación de hechos adicionales

Buscar opiniones

No solicitar hechos sino opiniones o valores pertinentes a los problemas

Dar información

Entregar hechos en relacion con los problemas, o relatos de la propia experiencia

\section{Elaborar}

Desarrollar significados más claros o adicionales, o propor-
Iniciar ideas o cambios cuando no son necesarios

Pedir más información cuando ya se ha entregado la suficiente

Buscar opiniones cuando los hechos mismos son relevantes

Oscurecer el problema entregando más información de la necesaria

Exigir más elaboración cuando el problema está claro

12 Johnson, L. C., op. cit., págs. 212-213.

13 Tschorne, P., La Dinámica de grupo aplicada al Trabajo Social, Ed. Obelisco, Barcelona, 1990, pág. 20. 


\begin{tabular}{|c|c|c|}
\hline $\begin{array}{l}\text { Inhibidores por } \\
\text { escasez de } \\
\text { comportamiento }\end{array}$ & Facilitadores & $\begin{array}{l}\text { Inhibidores por } \\
\text { exceso de } \\
\text { comportamiento }\end{array}$ \\
\hline
\end{tabular}

cionar razones o deduc-

ciones

Coordinar

No coordinar cuando Mostrar relaciones enes necesario

No dar la orientación necesaria tre ideas y hechos

Orientar

Definir la dirección de los objetivos

Evaluar

No evaluar, o evaluar Proveer criterios de muy poco rendimiento y someter a medición el progreso del grupo

\section{Estimular}

Aceptar la apatía o no Estimuar al grupo para participación que alcance sus objetivos. Despertar mayor y mejor participación

Forzar relaciones entre ideas y hechos

Orientar de manera excesivamente determinada y restrictiva

Evaluar demasiado

Excesiva estimulación, con el efecto de una actividad no productiva

Además del liderazgo de tarea, cualquier grupo efectivo necesita también el llamado liderazgo de mantenimiento que, entre otros autores ha sido estudiado profundamente por David y Frank Johnson ${ }^{14}$.

Este liderazgo se centra fundamentalmente en la facilitación de las relaciones de participación, en la atención al clima emocional y a los sentimientos, en la fijación de pautas de comportamiento, en la creación de un clima de confianza y en el intento de dar una solución positiva a los conflictos interpersonales.

La tarea fundamental que incumbe al Trabajador Social como miembro y líder de grupo, se relaciona precisamente con el propósito de colaborar de forma activa a que sea dinámica y viva la interacción que tiene lugar en él, tanto en el proceso que conduce a su formación y consolidación, como en las etapas posteriores en que realizan sus actividades específicas. El sentido y alcance de su aportación a esta finalidad básica ha sido resumido por Louise Johnson en unas propuestas concretas que muy sucintamente pueden resumirse así:

1. Aceptación de los miembros del grupo con sus ideas, actitudes y sentimientos. Esta disponibilidad no sólo hará conscientes a los miembros del grupo del papel que desempeña el Trabajador Social en la

14 Johnson, D. W. y Johnson, F. P., Joining Together: Group Theory and Group Skill, Englewood Cliffs, Nueva York, Prentice Hall, 1975. 
configuración de un clima favorable de convivencia y de trabajo, sino que les empujará a desempeñar su tarea con mayor confianza y eficacia.

2. ${ }^{\circ} \quad$ Utilización de la relación interpersonal para ayudar a los miembros del grupo a encontrar fines comunes, al mismo tiempo que posibilita que vayan ellos mismos desarrollando habilidades interaccionales y profundizando su intercomunicación.

3. Apoyo y estímulo en el desempeño de implicarse activamente en las actividades del grupo y en sus decisiones, al mismo tiempo que se contribuye a esclarecer el proceso que se está viviendo en su seno y los posibles modos de modificarlo para cumplir más eficazmente sus funciones.

4. ${ }^{\circ} \quad$ Limitación de actividades o conductas que pueden representar un obstáculo o constituyan una amenaza para las relaciones interpersonales de los miembros del grupo.

5. Orientación razonada de los procesos de discusión y de los modos de afrontar los problemas e intentar buscar soluciones.

6. Oferta de ayuda y colaboración activa en la reducción de tensiones, ansiedades, conflictos o miedos que pudieran interferir en el funcionamiento eficaz del grupo.

7. Capacidad de hacer inteligible la función de la institución y la labor del Trabajador Social en relación a la tarea del grupo.

8. Atención permanente a las vicisitudes internas del grupo, intentando comprender lo que acontece en él y por qué acontece.

9. Finalmente planificación de sus propias tareas de modo que el grupo pueda cumplir sus funciones específicas, teniendo presente el estadio de desarrollo que está viviendo y la situación personal en cada uno de sus miembros ${ }^{15}$.

Por otra parte, a propósito del problema de la cohesión de los grupos, deben tenerse presentes también otros factores relacionados, por ejemplo, con el número de individuos que componen el grupo, su edad y mentalidad, las diferencias de caracteres, a las motivaciones inconscientes que los mueven o bien el interés común consciente que une a sus miembros.

Limitándonos aquí a este último factor de cohesión, es importante hacer referencia al concepto de valor, no ya desde una perspectiva ontológica o ética, sino desde el punto de vista de la teoría de la acción social.

Kluckhohn, por ejemplo, después de distinguir claramente entre lo que es, lo que se desea de hecho y lo que debería desearse, afirma que el valor es una concepción, explícita o implícita, propia de un individuo o característica de un grupo, acerca de lo deseable, lo que 
influye sobre la selección de los modos, medios y fines de acción accesibles ${ }^{16}$.

La definición, pues, engloba los principales elementos constituyentes del valor: el cognoscitivo (concepción), el afectivo (lo deseable) y el conativo (selección de medios y fines de acción).

En el caso del Trabajo Social esta atención a los fines específicos de su actividad se concreta en la opción por unos determinados valores que aspira a ver hechos realidad en las relaciones sociales, en las instituciones o en determinados ámbitos concretos de convivencia, a fin de que la calidad de vida a que quiere colaborar no sea una pura abstracción teórica o conceptual. Esto obliga, ante todo, a intentar hacer efectivos tales valores en los propios grupos de trabajo, en su dinámica interna y en su actuación social, esto es, hacer de ellos la clave última de sus motivaciones conscientes, de sus actitudes y comportamientos.

Evidentemente, esta opción consciente y compartida por unos determinados valores, que comprometen radicalmente a las personas, suponen en cada una de ellas en particular, y en la dinámica general del grupo, un grado de madurez que no siempre se alcanza.

Criterios básicos de la misma serían, entre otros, los siguientes:

- La asertividad, esto es un conjunto de cualidades como la capacidad de decisión o de compromiso, el espíritu de iniciativa, que imprimen a una vida una dirección precisa. Significa también la capacidad de conocer las propias exigencias o posibilidades y de expresarlas de forma adecuada en el propio ambiente.

- La extensión del sentido de sí mismo o, como dice Allport ${ }^{17}$, la superación de las fronteras del yo, la ampliación de los horizontes personales y el interés activo por los demás.

- La estructuración estable de las propias ideas, que se manifiesta en dos criterios básicos, uno dirigido hacia el exterior, esto es, la percepción objetiva de la realidad, y el otro hacia el interior, es decir, el conocimiento objetivo de sí mismo o autoobjetivación.

- La estabilidad y seguridad emotiva, de modo que se llegue a un equilibrio entre la esfera racional y la emocional, que son los dos polos en torno a los cuales gira la vida psíquica del hombre.

16 Kluckhohn, «Values and Value Orientation in a Theory of Action», en Toward a General Theory of Action, a cargo de Parsons, T. y Shils, E., Cambridge, 1951.

17 Allport, G., ¿Qué es la personalidad?, Siglo XXI, Buenos Aires, 1976, pág. 111. Una exposición detallada de su punto de vista puede verse en Ronco, A., «Personalità e educazione secondo G. W. Allport», Orient. Pedag., xvi, 1969 , págs. 782 y ss. 
En los grupos de Trabajo Social este doble tipo de funciones constituyen una referencia fundamental para la realización de una labor de liderazgo efectiva. Una síntesis de ambas formas de actuación sería lo que se ha llamado liderazgo centrado en el grupo, que combina la preocupación por la eficiencia real de las actividades y tareas que le son propias con la satisfacción de las necesidades y aspiraciones de los miembros y su intercomunicación efectiva.

Esto obliga a tener presentes los distintos estilos de liderazgo y los efectos que cada uno de ellos tiene sobre el comportamiento del grupo y de sus miembros, tal como hicieron, por ejemplo, $\mathrm{K}$. Lewin y sus colaboradores estudiando distintos climas grupales en relación con los líderes que tenían una incidencia directa en ellos ${ }^{18}$.

En un rol de liderazgo autoritario el líder determina por completo el modo de proceder del grupo, impone técnicas y actividades, diseña por anticipado el plan a desarrollar, no explica las razones que motivan sus decisiones, manifiesta una actitud impersonal y distante y se muestra subjetivo en la valoración de la labor de los miembros del grupo, con el cual no se compromete nunca totalmente.

En el liderazgo democrático, en cambio, el líder permite que el grupo determine los planteamientos generales, esboce las etapas que conducen al objetivo propuesto o sugiera procedimientos alternativos, favoreciendo también las discusiones del grupo y buscando el consenso de éste, a la vez que participa directamente en las tareas que se realizan y en su atmósfera emocional.

Finalmente el líder laissez-faire desempeña una función más pasiva, no participando en las actividades grupales y otorgando al grupo completa libertad para tomar sus propias decisiones. Tan sólo está presente para proporcionar informaciones y material cuando sean solicitados.

Lo fundamental de estos estudios consistió en permitir establecer que cada tipo particular de liderazgo determina estilos distintos de comportamiento o atmósferas de grupo. Pero hay que tener presente que nunca se trata de tipos puros o abstractos, por lo que en la compleja realidad grupal sólo puede hablarse de formas predominantes de liderazgo.

En un liderazgo democrático las relaciones entre los miembros son más solidarias, aparecen mayores diferencias personales y existe una mayor estabilidad, un sentido más acusado de responsabilidad y de compromiso.

El liderazgo autoritario, en cambio, provoca dos tipos de relaciones excluyentes: una agresiva y de rebeldía frente al líder que favore-

18 Lewin, K., Lippitt, R. y White, L., «Climas grupales en distintos tipos de liderazgo», en Cartwright, D. y Zander (comp.), Dinámica de grupos, investigación y teoría, Tillas, México, 1971. 
ce lógicamente la unión de miembros entre sí, y otra reacción apática como resultado de la frustración que se genera.

Finalmente, en un liderazgo laissez-faire es fácil constatar la falta de incentivo y la ausencia de técnicas necesarias para llegar a decisiones y planteamientos de tipo cooperativo, siendo evidente la tendencia a la dispersión del grupo y la consiguiente ineficacia de sus actuaciones.

Hay que tener presente, por otra parte, el fondo de verdad que se contiene en una observación que hace Marvin Shaw con el fin de evitar planteamientos simplistas. Según él, la situación y la función particulares del grupo determinan últimamente cuál ha de ser el estilo de liderazgo que debe prevalecer. Es evidente que es más fácil ser un líder autocrático que se limita a dar órdenes que un líder democrático que sabe canalizar las habilidades de los miembros del grupo de forma eficaz. Sin embargo, la conducta del líder no sólo depende de sus cualidades o de su actitud personal sino también de las características de la situación ${ }^{19}$.

En este contexto entiendo que el modo de influencia que pueden tener las conductas del líder sobre la motivación intrínseca de los miembros de un grupo, se ilustra teniendo presente el modelo que ofrece el profesor Tetrick y que es el resultado de una serie de estudios empíricos concluidos en 1989. Tras distinguir entre comportamientos que se limitan a transmitir información y comportamientos que condicionan o controlar la conducta de otros, sintetiza su posición en el esquema siguiente:

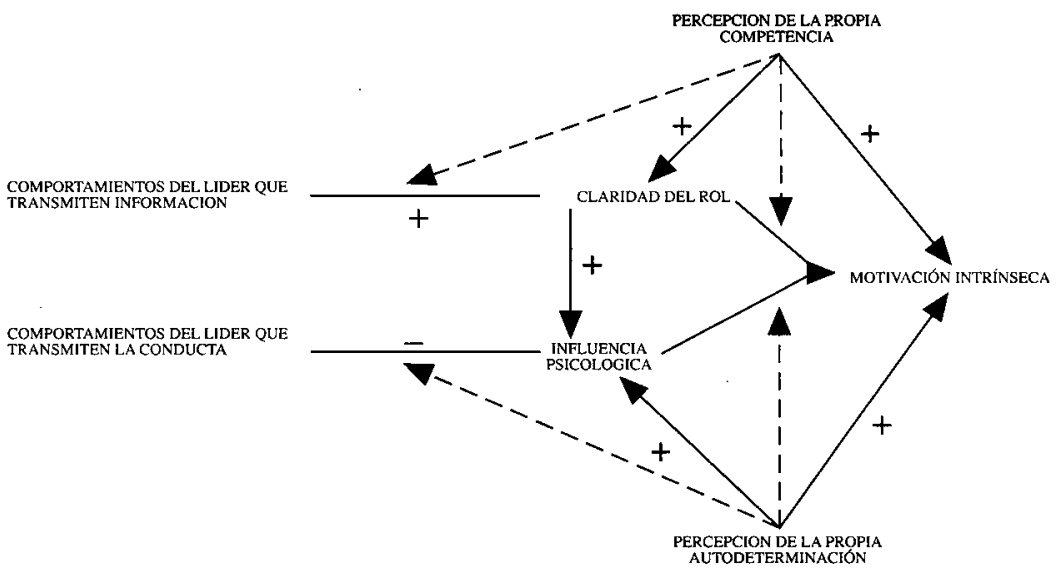

De acuerdo con este modelo, la percepción que tienen los miembros del grupo de la influencia que ejercen sobre ellos los comportamientos de líder, no puede separarse de la percepción que tengan de su propia competencia y capacidad de autodeterminación.

19 Shaw, M., op. cit., págs. 320-322. 
La función del líder, en suma, sería ofrecer información a los demás, de modo que contribuya a clarificar lo que debe hacerse y muestre cómo debería realizar las tareas concretas que le asigne el grupo. El propósito último será siempre conseguir la motivación intrínseca de los miembros del grupo, en cuanto a partir de ella se facilita la realización de sus objetivos y metas ${ }^{20}$.

\subsubsection{Las situaciones de conflicto y el liderazgo de grupo}

Una última cuestión relacionada con el liderazgo de grupo del Trabajador Social es la que se refiere a su papel en las situaciones de conflicto que pueden presentarse en el grupo.

Desde la perspectiva que aquí nos interesa, y siguiendo las observaciones que han venido haciendo por parte de diversos autores ${ }^{21}$, entiendo que es útil distinguir entre el conflicto propiamente dicho y la situación de crisis que pueden afectar a cualquier sistema social. Lo fundamental en el conflicto es que se trata de un enfrentamiento entre diversas opciones, que manifiestan una intención de rechazo mutuo. En este sentido, el conflicto no se entiende ya en un sentido meramente figurado (como cuando se habla, por ejemplo, de conflicto de deberes), o como una simple rivalidad o desacuerdo, sino que da lugar a planteamientos que se excluyen y a una incompatibilidad real de intereses.

La noción de crisis es más general o abarcadora y está ligada a las alteraciones o rupturas que puede provocar el desarrollo mismo de la sociedad y los cambios que lo acompañan. En la medida en que un grupo humano sea capaz de renovarse y adaptarse a las nuevas situaciones podrá conservar su identidad y desplegar sus posibilidades de acción, superando los momentos críticos que van planteándose ${ }^{22}$.

Es evidente que no todo cambio da lugar necesariamente a una crisis, sobre todo si se realiza de forma regular y casi imperceptible. $\mathrm{La}$

20 Tetrick, L. E., «The Motivating Potential of Leader Behaviors: A Comparison of Two Models», Journal of Applied Social Psychology, 19,11, 1989, págs. 947-958; entiendo que la idea central que desarrolla el autor en este trabajo de investigación se resume en esta afirmación introductoria: «Psychological influence increases intrinsic motivation but role clarity influences intrinsic motivation only indirectly throuhg its influence on psychological influence».

21 Ver por ejemplo los estudios que se publican en el núm. 5 de la revista Communications, 1976, en especial el trabajo de Freund, J., «Sur deux catégories de la dinamique polémogène», págs. 101-112.

22 Morin, E., «Pour une crisologie», Communications, núm. 5, 1976, págs. 149 y ss.; el autor observa que para tener un concepto adecuado de crisis, yendo más allá de la idea de perturbación o ruptura de equilibrio, sería preciso «concevoir la société comme système capable d'avoir des crises, c'est-à-dire poser trois ordres de principes, le premier sistémique, le second cybernétique, le troisème néguetropique, sans quoi la théorie de la société est insuffisante et la notion de crise inconcevable». 
auténtica crisis, que puede degenerar en conflicto, se plantea como consecuencia de una modificación súbita que lleva consigo un estado de desequilibrio y de incertidumbre. La alteración que ello provoca puede ser de dos clases: o bien es el resultado de la intervención de factores externos, o está producida por el desarrollo mismo de un proceso que, una vez superado cierto umbral, crea un estado de ruptura... En otras ocasiones la crisis manifiesta un desajuste entre la aceleración de algunos aspectos de la evolución de un grupo y el retraso de otros, lo cual provoca una ruptura en el ritmo normal de cambio.

En cualquier caso, lo fundamental consiste en discernir las causas y el alcance de la situación de crisis o de conflicto que se está viviendo en el grupo, y en saber tomar las decisiones adecuadas a la nueva situación que se plantea.

En los grupos de Trabajo Social, se mantiene claramente esta ambivalencia de la crisis y del conflicto. Para que su desenlace sea positivo se requiere un análisis ponderado de las experiencias, puntos de vista, interacciones y procesos de comunicación que confluyen en el grupo.

Uno de los signos indicadores de la existencia de conflictos no resueltos en el seno del grupo, o de deterioro en el proceso grupal, es la aparición frecuente en él de los llamados roles disfuncionales, esto es intentos de satisfacer necesidades individuales sin relación directa alguna con la tarea del grupo.

Entre estos roles hay que hacer referencia, por ejemplo, a los siguientes:

A) Rol de obstructor, en cuanto expresa desacuerdos sin razones que lo justifiquen, o sea retrotrae a problemas ya resueltos.

B) Rol de buscador de reconocimiento cuya intención última es llamar la atención sobre sí mismo o alimentar una actitud narcisista.

C) Rol llamado de confesante, esto es, de manifestación de sentimientos personales que no tienen vinculación con la tarea explícita, confundiéndose el grupo de trabajo con un grupo terapéutico.

D) Rol de descomprometido con los procesos grupales y que hace alarde de ello, mostrándose lejano y autosuficiente.

E) Rol de dominador, o que usa la conducta agresiva como medio para imponer sus criterios o ideas, impidiendo la participación activa de otros.

F) Rol de buscador de ayuda, es decir que, intenta lograr una respuesta de simpatía a través de expresiones de inseguridad, confusión personal o depreciación de sí mismos.

$\mathrm{G})$ Rol de defensor de intereses especiales, que busca la defensa de intereses ajenos al grupo y a sus tareas específicas ${ }^{23}$.

${ }^{23}$ Cf. Romero, R., Grupo. Objeto y Teoría, vol. II, Lugar Ed., Buenos Aires, 1992, págs. 51-53. 
En otras ocasiones se va incluso más allá, afirmándose que en todo grupo el conflicto aparece como una consecuencia inevitable de la existencia de los llamados grupos superpuestos y que, como ha expuesto Thelen, son fundamentalmente éstos:

1. Subgrupos efectivos de pertenencia o grupos internos en los que puede constatarse un vínculo privilegiado de afinidades o amistad entre varios de sus integrantes. Los participantes de estos subgrupos perciben a la totalidad del grupo desde la perspectiva que les ofrece esta situación peculiar que viven y que se superpone lógicamente al grupo real.

2. Subgrupo de arrastre que se refiere al intento de establecer en todo grupo la reiteración o repetición de vínculos aprendidos en el pasado, es decir vínculos gratificantes o frustrantes específicamente aprendidos en el grupo familiar de origen.

3. Otros subgrupos que, con diversas denominaciones, responden ya sea a imágenes pre-conscientes o no plenamente conscientes de sus integrantes y que pueden constituirse en fuente posible de conflictos en el grupo.

Lo fundamental del planteamiento de Thelen es que el origen de una parálisis grupal no es el conflicto por sí mismo, sino la superposición de elementos no controlados en él. La labor de un líder de grupo consiste fundamentalmente en descubrir y determinar estos factores que sólo implícitamente o de forma poco consciente se interfieren en la dinámica del grupo.

Refiriéndonos de forma más explícita a la labor de líder del Trabajador Social en relación con el encauzamiento y control del conflicto en los pequeños grupos, entiendo que las ayudas fundamentales que puede aportar son, resumiendo muy abreviadamente la exposición que hace Louise Johnson, las siguientes:

- Definir el conflicto, no como un problema de una sola persona, sino como una realidad que afecta a todo el grupo.

- Escuchar el punto de vista de cada una de las personas, intentando determinar sus coincidencias y divergencias.

- Evitar soluciones del tipo vencedores y vencidos.

- Analizar las causas reales que han conducido al conflicto, desenmascarando aquellas que puedan estar encubiertas por razones o circunstancias de diversa naturaleza.

- Tomar iniciativas en orden a hacer que prevalezcan las actitudes de cooperación sobre el clima de rivalidad o competencia.

Tras exponer y razonar estas ideas, la autora observa textualmente que el reconocimiento del conflicto es el primer paso hacia su posi- 
ble solución. Cuando el conflicto no es reconocido o aceptado, puede ser destructivo de la interacción específica del grupo ${ }^{24}$.

\section{REFLEXION FINAL}

A modo de reflexión final, y teniendo presentes las líneas generales de la exposición que antecede, a propósito de la labor del Trabajador Social como miembro o líder de grupo, entiendo que es importante reiterar la idea que hacíamos inicialmente en el sentido de que cada grupo en particular tiene su dinámica propia, aunque puede hablarse de unas características generales comunes a cualquier grupo.

Un ejemplo que ilustra el modo de aplicar estos criterios puede ser, entre otros, el estudio publicado recientemente por la psicóloga y psicoterapeuta Lola Arrieta ${ }^{25}$ a propósito de los procesos de reinserción de grupos marginados. Al analizar las fases que atraviesa un grupo de esta naturaleza, va resaltando el alcance y significado que tienen las intervenciones del Trabajador Social o del animador en cada una de ellas, desde el momento inicial de la acogida hasta el desenlace final del proceso.

En éstas y otras situaciones, la labor del trabajador social, como miembro, animador o líder de grupo, no obedece a unas pautas fijas, sino que supone un esfuerzo permanente de creatividad para adaptarse a los diversos ámbitos y situaciones en que operan los grupos, a sus finalidades específicas, a su propia historia evolutiva y a los múltiples factores que condicionan las relaciones internas de sus miembros y sus modos de actuación.

En suma, y como conclusión general de esta exposición, quiero manifestar mi convicción de que también aquí, como en otros campos de intervención del Trabajador Social, es necesario permanecer en contacto directo con las realidades y con las personas y no limitarse únicamente a actuar guiándose por los principios generales o por las ideas que nos hacemos de ellas.

24 L. Johnson, op. cit., pág. 212.

25 Arrieta Olmedo, L., «Los procesos de reinserción de los grupos marginados», Documentación Social, núm. 81, 1990. 